\title{
HYDROPHILIZATION OF POLYESTER TEXTILES BY NONTHERMAL PLASMA
}

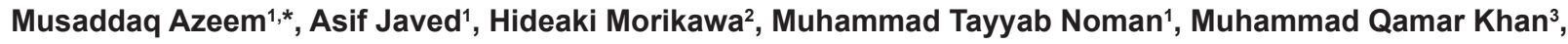 \\ Muhammad Shahid ${ }^{1}$, Jakub Wiener ${ }^{1}$ \\ 1 Technical University of Liberec, Faculty of Textile Engineering, Department of Material Engineering, Studentská 1402/2, 461 17, Liberec 1, Czech Republic \\ 2 Faculty of Textile Science and Technology, Shinshu University, 3-15-1 Tokida, Ueda City, Nagano 386-856, Japan \\ 3 Faculty of Engineering and Technology, National Textile University Karachi campus, 2/1 Sector 30 Karachi, Sindh 74900, Pakistan \\ ${ }^{*}$ Corresponding author e-mail: musaddaqazeem@yahoo.com
}

\begin{abstract}
:
Polyester is a popular class of material used in material engineering. With its $0.4 \%$ moisture regain, polyethylene terephthalate (PET) is classified as highly hydrophobic, which originates from its lack of polar groups on its backbone. This study used a parallel-plate nonthermal plasma dielectric barrier discharge system operating at medium pressure in dry air and nitrogen $\left(N_{2}\right)$ to alter the surface properties of PET fabrics to increase their hydrophilic capabilities. Water contact angle, atomic force microscopy (AFM), and X-ray photoelectron spectroscopy (XPS) were utilized to analyze any effect from the plasma treatment. The wettability analysis revealed a reduction in the contact angle of more than $80 \%$ within 5 min for both discharges. Scanning electron microscopy analysis showed no microscopic damage to the fiber structure, guaranteeing that the fabrics' structural integrity was preserved after treatment. AFM analysis showed an increase in the nanometer roughness, which was considered beneficial because it increased the total surface area, further increasing the hydrophilic capacity. XPS analysis revealed a sharp increase in the presence of polar functional groups, indicating that the induced surface changes are mostly chemical in nature. Comparing that of untreated fabrics to treated fabrics, a Increase in water absorption capacity was observed for airtreated and $\mathrm{N}_{2}$-treated fabrics, when these fabrics were used immediately after plasma exposure.
\end{abstract}

\section{Keywords:}

$D B D$, medium plasma treatments, AFM, PET, hydrophilicity, XPS, wettability

\section{Introduction}

Polyethylene terephthalate (PET), commonly referred to as polyester, is considered to be one of the most important polymers in the textile industry because of its compelling characteristics, such as high strength, formability, dimensional and thermal stability, degree of hardness, and excellent chemical properties $[1,2]$. Unfortunately, PET has a very hydrophobic nature with only $0.4 \%$ moisture regain [3]. This makes it a very challenging material for aqueous postproduction processes, such as dyeing. PET's hydrophobic nature is also problematic for hydrophilic applications, a process that requires textiles to absorb and transport liquids. To overcome this problem, chemical modifications [4] have been studied to make PET fabrics more hydrophilic. These modifications, generally referred to as moisture management, are very depending on the type and structure of the fibers or fabric, the finishing that is applied to the material, and other possible postproduction processes [3, 5-7]. As chemical modifications often compromise the structural integrity of PET meshes, numerous alternative methods have been investigated. Nonthermal plasma treatment is one of the most promising surface modification technologies that has been reported over last decade [8, 9]; plasma technology recently gained interest because of its improved capacity to effectively change the wettability, lack of solvents, and low power consumption [10-12]. These modifications also tend to change the textile adhesion properties [13]. DBD atmospheric pressure plasma systems are of particular interest, as they do not require expensive vacuum equipment, allowing for straightforward, in-line implementation in industrial processes [14-19]. It is well-known that plasma treatments (when operated under optimal conditions, using inert gases such as air or nitrogen) only affect the first few subsurface atomic layers while preserving the overall bulk properties [8].

The experiments performed in this work were done with a medium pressure system, which combines the benefits of low pressure and atmospheric pressure systems: higher levels of control and reproducibility with large plasma volumes and a reduced cost because of less complex vacuum equipment, respectively [20]. Furthermore, it was shown that a lower treatment pressure was more efficient in transporting the plasma active species throughout fibrous meshes than at elevated pressure, thus provides more homogeneous treatment of these porous structures. In this study, nonwoven PET sheets were treated with parallel-plate plasma dielectric barrier discharge (DBD) at medium pressure $(5.0 \mathrm{kPa})$ in dry air and nitrogen plasma. The following tests and techniques were used to characterize the untreated and plasma-treated nonwoven polyester fabrics: scanning electron microscopy 
(SEM), X-ray photoelectron spectroscopy, water contact angle (WCA), atomic force microscopy, water absorption capacity test, and wettability test.

\section{Materials and methods}

The $100 \%$ nonwoven polyester fabrics were acquired from JX Nippon ANCI Corporation, Japan. Small samples were cut into small pieces of $10 \mathrm{~mm} \times 30 \mathrm{~mm}$. Before processing, all samples were exposed to ultrasonic treatment in ethanol (VWR, 90\% pure) for $20 \mathrm{~min}$ to remove any superficial contamination. After ultrasonic treatment, samples were dried at room temperature and used as such (Table 1).
Nonwoven machines do not make the uniform fabric so the orientation is not the same between machine direction (MD) and cross direction (CD); hence, the tensile strength is measured in both directions.

\subsection{Plasma treatment with $D B D$ reactor}

All plasma treatments reported in this article were done on a parallel-plate DBD system (see Figure 1) that was operated at medium pressure, as described earlier [20]. The system consists of two copper electrodes $(\varnothing: 50 \mathrm{~mm}$ ) covered with glass plates. The upper electrode (attached to $50 \mathrm{kHz} A C$ voltages) and the lower electrode (attached to $50 \Omega$ or $10 \mathrm{nF}$ capacitor) were separated by a $35-\mathrm{mm}$ gap. A single fabric
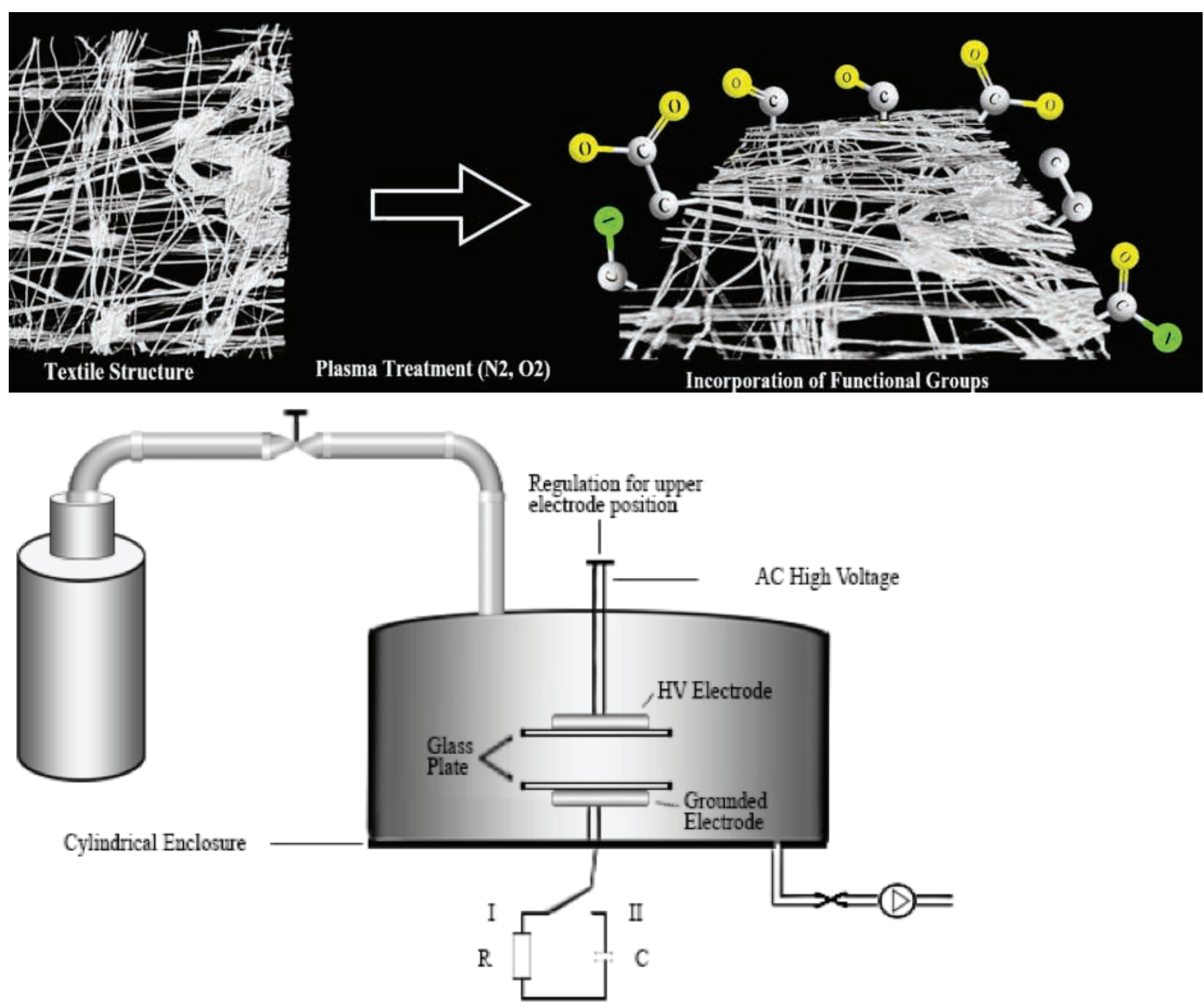

Figure 1. Set-up of the DBD discharge.

Table 1. Properties of polyester fabric evaluated in this study

\begin{tabular}{|c|c|c|c|c|c|c|}
\hline \multirow{2}{*}{ Density $\left(\mathbf{g} / \mathbf{m}^{3}\right)$} & \multirow{2}{*}{$\begin{array}{c}\text { Thickness } \\
(\mathbf{m m})\end{array}$} & $\begin{array}{c}\text { Area density } \\
\left(\mathbf{g} / \mathbf{m}^{2}\right)\end{array}$ & \multicolumn{2}{|c|}{$\begin{array}{c}\text { Tensile strength } \\
\mathbf{( N / 5 0 ~} \mathbf{~ m m})\end{array}$} & \multirow{2}{*}{ Contact angle $\left(^{\circ}\right)$} & Porosity (\%) \\
\cline { 3 - 5 } & & 10 & MD & CD & 118 & 47.4 \\
\hline $1.33-\sim 1.42$ & 0.083 & 25 & 18 & \\
\hline
\end{tabular}


sample was placed onto the lower electrode. After that, the plasma reactor was pumped to less than $0.5 \mathrm{kPa}$ using a rotary vane pump. Subsequently, the system was filled with dry air or nitrogen at 3 standard liter per minute (s/m) up to $50 \mathrm{kPa}$; the plasma reactor was flushed at $3 \mathrm{slm}$ at said pressure for $3 \mathrm{~min}$ to achieve a reproducible discharge gas composition. After that, the pressure in the plasma reactor was reduced to $5.0 \mathrm{kPa}$ and $1 \mathrm{slm}$ and used to perform the treatments between 0 and 9 min at fixed intervals [20].

\subsection{Electrical properties of the DBD}

To analyze the type of generated discharge, the currentvoltage waveform at $5 \mathrm{kPa}$ was recorded. The applied voltage was sinusoidal, whereas the discharge current consisted of numerous short peaks. Each peak corresponded to a series of micro discharges, and therefore, one can conclude that the used DBD operated in the filamentary mode. The discharge power of each treatment was calculated from the voltage change plots (Lissajous; see Figure 2) using the following formula:

$$
\text { Power }=2\left\lfloor\left(U_{\max } \times Q_{\min }\right)-\left(U_{\min } \times Q_{\max }\right)\right\rfloor \times \text { frequency } \times 10^{-6}
$$

where $U$ is the voltage in $\mathrm{kV}$ and $Q$ is the charge in $\mathrm{nC}$. The discharge power, as calculated from Eq. (1), was $4.55 \mathrm{~W}$ for air, while $3.44 \mathrm{~W}$ was recorded for nitrogen. The energy density of each treatment was determined through Eq. (2):

$$
\text { Energy density }\left(\mathrm{J} / \mathrm{cm}^{2}\right)=\frac{t(s) \times P(W)}{S}
$$

where $t$ is the plasma exposure time, $P$ is the plasma power, and $S$ is the area of the electrodes.

The area of the electrodes was constant for all treatments and was equal to $12.56 \mathrm{~cm}^{2}$. For all plasma discharges, different samples were prepared while varying the plasma exposure time (treatment times varied between $0 \mathrm{~s}$ and 9 min for dry air and nitrogen treatments).

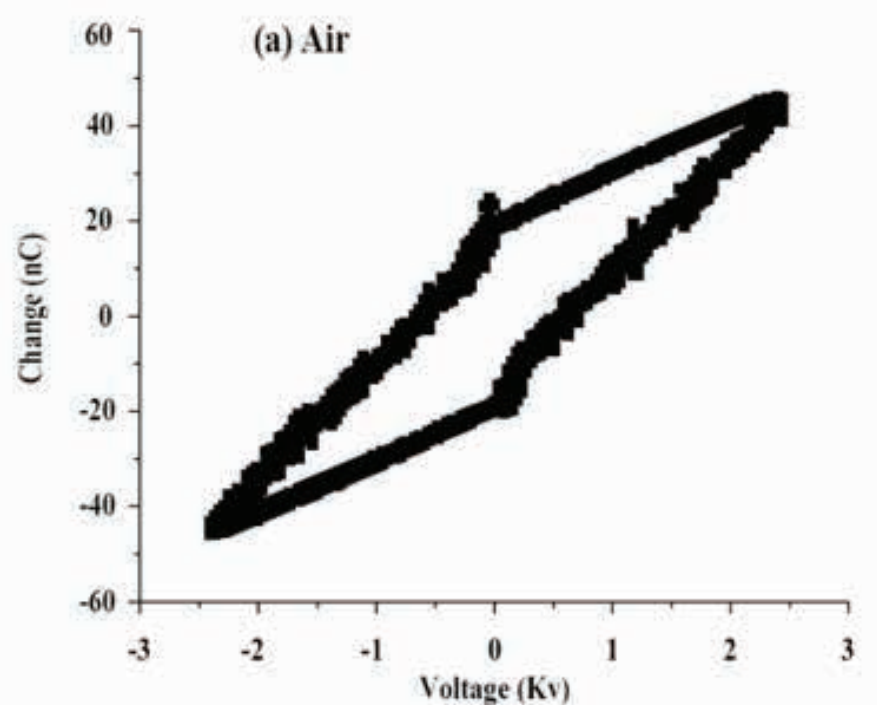

\subsection{SEM}

The structure of the untreated and plasma-treated nonwoven polyester fabrics was analyzed by SEM (JSM-6010 PLUS/LV; JEOL, Japan). SEM images were obtained with an accelerating voltage of $7 \mathrm{kV}$ at a working distance of $10 \mathrm{~mm}$ after samples were covered with a gold coating, making use of a sputter coater (JFC-1300 autofine coater; JEOL).

\subsection{Contact angle}

Plasma-treated samples were subjected to static WCA measurements within 5 min after treatment, using a Krüss Easy Drop optical system (Krüss $\mathrm{GmbH}$, Hamburg, Germany). A 2- $\mu$ l drop volume was used, and the contact angles were automatically measured within $3 \mathrm{~s}$ using the Laplace-Young curve fitting procedure. Each contact angle value described in this article is the mean of seven random measurements over a single sample with standard deviations consistently varying between $0.1^{\circ}$ and $2.5^{\circ}$.

\subsection{AFM}

Possible changes in surface topography were quantified using an XE-70 AFM system (Park Systems, Suwon, South Korea). Micrographs of $15 \mu \mathrm{m} \times 15 \mu \mathrm{m}$ were recorded in a noncontact mode using a silicon-based cantilever (PPP-NCHR; Nanosensors ${ }^{\mathrm{TM}}$, Neuchâtel, Switzerland). Micrographs were inspected using the included XEP processing software (V1.8.0) and were subjected to an $X-Y$ plane autofit procedure before roughness determination. For each condition, two different samples were analyzed at three random locations per sample.

\subsection{XPS}

XPS surface analysis was performed on a PHI 5000 Versaprobe II system (ULVAC-Physical Electronics, Chigasaki, Japan) equipped with a monochromatic Al Ka X-ray source $(h v=1,486.6 \mathrm{eV})$ operated at $25 \mathrm{~W}$. The vacuum in the main chamber was kept at below $1 \times 10^{-6} \mathrm{~Pa}$ during measurements.

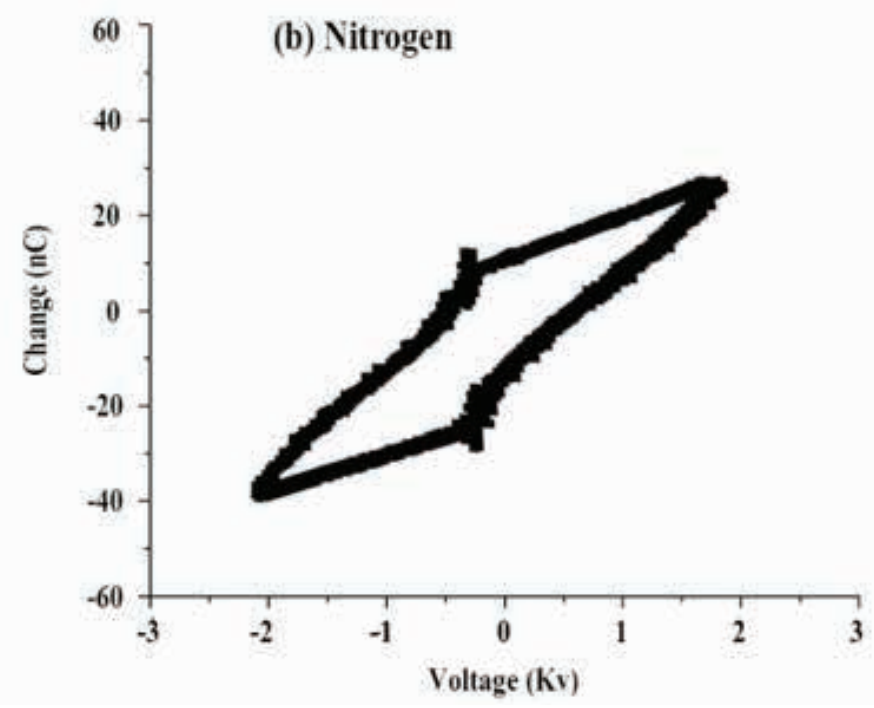

Figure 2. The Lissajous image at $4.55 \mathrm{~W}$ (dry air) and $3.44 \mathrm{~W}$ (nitrogen gas). 
Both the survey scans and the high-resolution spectra (C1s, O1s, and N1s) were recorded using pass energies of $187.85 \mathrm{eV}(\mathrm{eV}$ step $=0.8 \mathrm{eV})$ and $23.5 \mathrm{eV}(\mathrm{eV}$ step $=0.1 \mathrm{eV})$, respectively, with the hemispherical analyzer set at $45^{\circ}$ of the sample's normal. The atomic elemental composition was calculated from the survey spectra using the Multipak software (v 9.6.1). A spectrum calibration $(C-C=285.0 \mathrm{eV}$ ) was done before analysis, and an iterated Shirley background was applied to determine the elemental composition using the relative sensitivity factors supplied by the manufacturer. The curve peak fitting of the individual high-resolution peaks was also done using Multipak after applying a Savitzky-Golay smoothing procedure. The peaks were deconvoluted using Gaussian-Lorentzian peak shapes, keeping the FWHM (full width at half maxima) below $1.4 \mathrm{eV}$ and the $X^{2}$ below 2. For each condition, one sample was analyzed at four randomly distributed locations on the sample.

\subsection{Wettability}

\subsubsection{Water absorption capacity test}

This test was performed according to the China standard GB 21655.1Y2008. The $10 \mathrm{~cm} \times 10 \mathrm{~cm}$ samples were first weighed in dry condition and then immersed in distilled water for $5 \mathrm{~min}$. Next, each fabric sample was hung vertically until no water dripped from it within a $30 \mathrm{~s}$ period. After $30 \mathrm{~s}$, the sample was weighed again. The given formula was used to calculate the water absorption ratio:

Water absorption ratio $(\%)=\frac{\text { wet weight }- \text { dry weight }}{\text { dry weight }} \times 100$

\subsubsection{Wettability test}

The absorption rate of the samples with respect to time was measured according to the standard method AATCC 79. A droplet of $5 \mu \mathrm{l}$ was poured from $1 \mathrm{~cm}$ above the substrate onto the surface of the tested substrate. Wettability was measured as the total time it took for the reflection of the liquid surface to disappear. The shorter the absorption time, the better the wettability [21]. Wettability of polyester fabric was enhanced by plasma treatments and then after the washing, shorter wetting times were noted. Storing of the samples under atmospheric conditions for 6 months did not alter the wettability [22].

\section{Results and discussions}

\subsection{WCA}

After plasma treatment, the WCA of the samples was measured. Figure 3 describes the static contact angle measurements for both dry air and nitrogen plasma-treated polyester samples. To represent the contact angle as a function of energy density, the average power of air and nitrogen in discharge was calculated by Eq. (2). The contact angle of the plasma-treated samples showed a significant difference compared to that of the untreated polyester sample.
Looking at the impact of the treatment time on the measured values of WCA, one could assume that the WCA of the samples decreased to a minimum level and slightly increased after reaching an energy density of 14.92 and $11.21 \mathrm{~J} / \mathrm{cm}^{2}$ for air and nitrogen, respectively. Therefore, the $14.92 \mathrm{~J} / \mathrm{cm}^{2}$ (for air) and $11.21 \mathrm{~J} / \mathrm{cm}^{2}$ (for nitrogen) conditions were fixed for AFM, XPS, and wettability discussion sections. Increasing the exposure time to the plasma treatment initially resulted in a progressive increase in hydrophilicity of the nonwoven samples, but, after a certain amount of time, saturation occurred, which correlates with the literature [23].

The samples treated with oxygen plasma had lower contact angle values. Moreover, at $180 \mathrm{~s}$, the surfaces revealed a higher WCA value regardless of the type of gas used. Nitrogen plasma treatment, however, seemed to be less efficient in producing high hydrophilicity at the same level of treatment time. At $5 \mathrm{~J} /$ $\mathrm{cm}^{2}$ of energy density with oxygen and nitrogen plasma, the surface started to show a change in its hydrophilicity. From $10 \mathrm{~J} / \mathrm{cm}^{2}$, the hydrophilicity remained almost constant.

Figure 3 shows that the contact angle is, in a relative manner, inversely proportional to the energy density. With the increase in energy density, the contact angle decreased to a certain limit for both the untreated and plasma-treated samples. The WCA measured at $180 \mathrm{~s}$ of treatment time was the lowest with the energy density of 14.92 and $11.21 \mathrm{~J} / \mathrm{cm}^{2}$ for air and nitrogen, respectively, so it was used in all other measurements [24]. The graph of WCA is plotted as the function of energy density, permitting an impartial comparison. The graph demonstrates that the decrease in energy density is directly related to the WCA and results in advancing of chemical functionalities.

\subsection{SEM}

SEM was used to observe the alterations in the microscopic scale surface morphology. Figure 4 depicts the SEM images of the polyester nonwoven before and after plasma treatment. The SEM images showed the following: untreated samples exhibited a smooth surface with no distinct topography, whereas plasma-treated samples had some spots of distinct topography. The appearance of these bumps was further visualized by AFM.

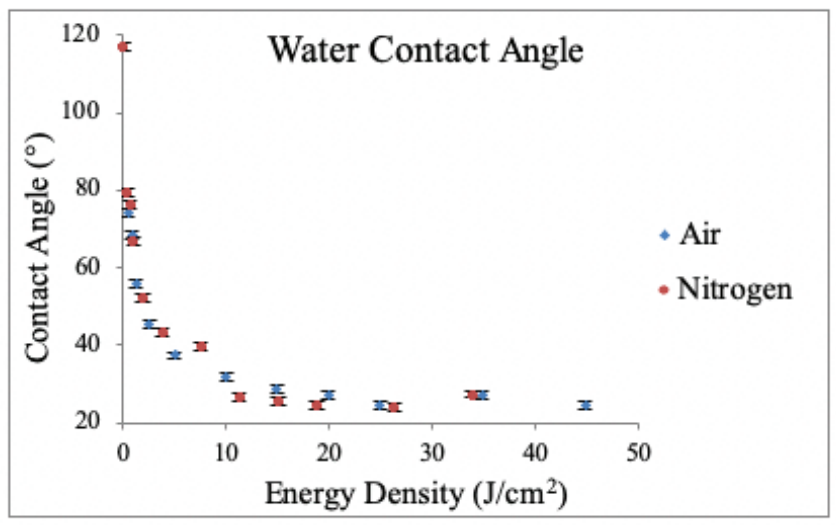

Figure 3. WCA measurements for plasma-activated nonwoven polyester fabric in the function of energy density for dry air and $\mathrm{N}_{2}$. 


\subsection{AFM imaging}

AFM images of the untreated and plasma-treated (air and nitrogen) PET textiles are shown in Figure 5. Compared with untreated samples, the plasma-treated (dry air and nitrogen) samples had shown the modified surfaces. The plasma-treated samples with dry air shown the highest value of the roughness, which can be linked to the reduced contact angle as compared to the nitrogen plasma-treated samples, as shown in Table 2.

The surface topography of the untreated sample appeared to be flatter in comparison to the plasma-treated samples (air and nitrogen). AFM images of plasma-treated showed small bumps on the surface whose size increased as the energy density increased. Changes in surface topography of the plasmatreated (air and nitrogen) samples were quantified by extracting the $R_{\text {rms }}$ (root mean square) surface roughness. Roughness is responsible for such behavior as the surface topography affects the WCA. This is possibly due to the polymer chains containing oxygen functionalities have turned toward bulk and that is why contact angle shows not much decreased after saturated region [25] as elaborated in Figure 3.

The $R_{\mathrm{rms}}(\mu \mathrm{m})$ roughness data were measured from many $1-\mu m^{2}$ AFM images [26].

Table 2. Roughness for untreated, dry air, and $\mathrm{N}_{2}$

\begin{tabular}{|c|c|c|c|}
\hline & Untreated & Air treated & $\mathbf{N}_{\mathbf{2}}$ treated \\
\hline$R_{\mathrm{rms}}(\mu \mathrm{m})$ & 0.017 & 0.599 & 0.307 \\
\hline $\mathrm{St}_{\mathrm{dev}}$ & \pm 0.010 & \pm 0.041 & \pm 0.056 \\
\hline
\end{tabular}

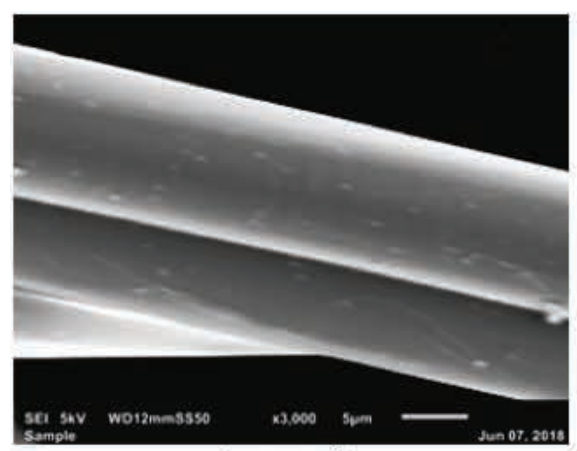

(untreated)

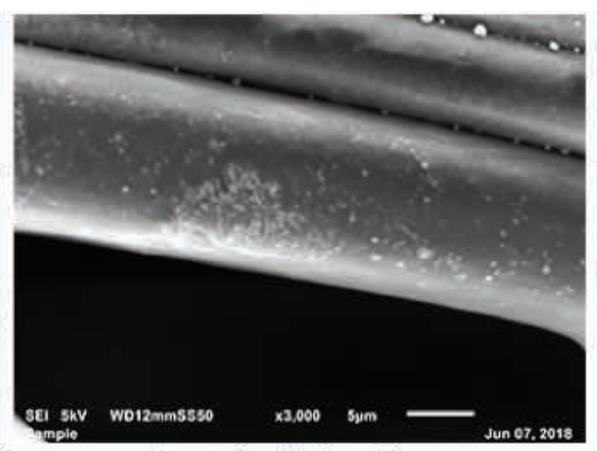

(treated with dry air)
To distinguish between the contribution of surface chemistry and surface topography to the total wettability, the chemistry of these functional groups is briefly described in the XPS section.

\subsection{XPS analysis}

A broad range of active species can be produced through a DBD discharge in the air, including ozone, nitrogen oxides, atomic oxygen, both neutral and meta-stable molecules, radicals, and ultraviolet radiation. The surface energy levels of polymers and textiles are improved directly when exposed to an air plasma, as oxygen-containing polar groups are being incorporated into the polymer surface. The major reactive species responsible for this oxygen addition [27] are believed to be atomic oxygen. The dissociation of $\mathrm{O}_{2}$ molecules by electron collision is what forms the atomic oxygen. Nonetheless, excitation and dissociation of nitrogen molecules may also contribute to several added reaction paths that can generate extra atomic oxygen [27, 28]. To better understand the nature of functional groups created by plasma treatment, the atomic composition of both untreated and plasma-treated samples was determined by XPS. The $\mathrm{O} / \mathrm{C}$ (oxygen/carbon) and N/C (nitrogen/carbon) atomic ratios for the untreated sample and two plasma-treated samples are shown in Table 3.

Table 3 shows the following observations for the O/C atomic ratio: an increase from 0.31 to 0.47 and 0.39 for plasma-treated air sample and plasma-treated nitrogen sample, respectively when compared with the untreated PET samples. An oxygen content of $23.4 \%$ was found in untreated (i.e., raw) samples, while an oxygen content of 31.5 and $26.5 \%$ was observed in air and nitrogen saturated, respectively, which indicates a

Figure 4. SEM images of untreated and plasma-treated (air and nitrogen) samples.

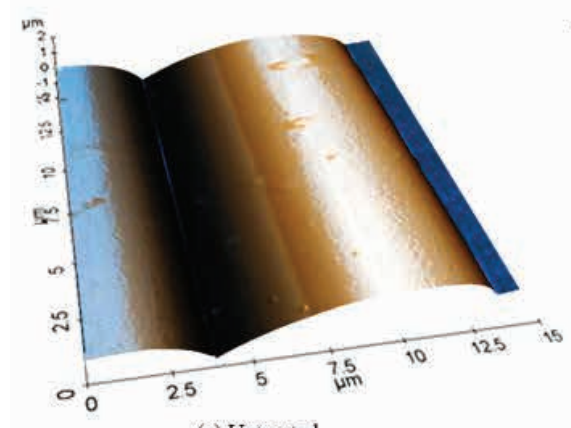

(a) Untreated

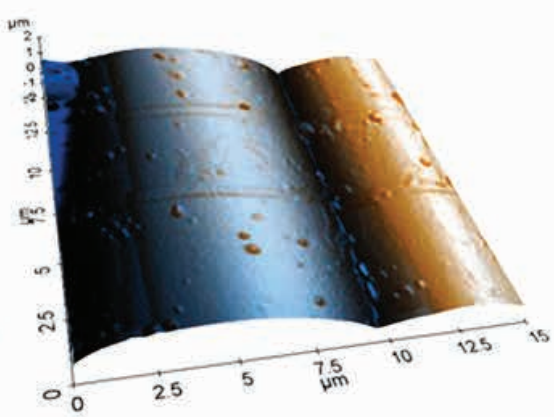

(b) Air Treated

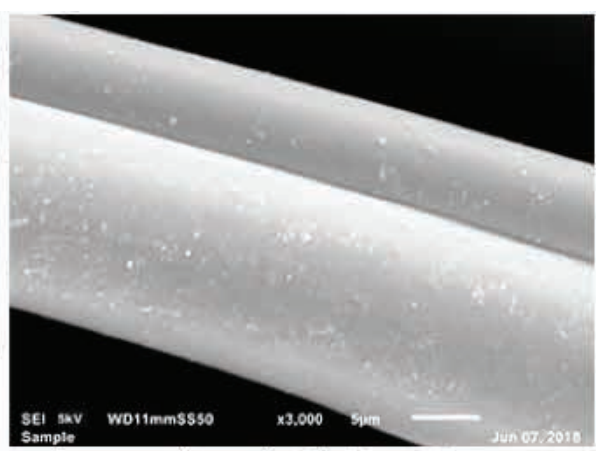

(treated with nitrogen)

Figure 5. Series of AFM images for (A) untreated, (B) dry air, and (C) $\mathrm{N}_{2}$ plasma-treated fabric samples. 
Table 3. Elemental composition and N/C, O/C, and $(\mathrm{N}+\mathrm{O}) / \mathrm{C}$ ratio

\begin{tabular}{|c|c|c|c|c|c|c|}
\hline Samples & C1s (at.\%) & N1s (at.\%) & O1s (at.\%) & N/C & O/C & $(\mathrm{N}+$ O)/C \\
\hline Untreated & $75.4 \pm 1.5$ & $1.0 \pm 0.5$ & $23.4 \pm 1.3$ & 0.013 & 0.310 & 0.323 \\
\hline Dry air & $66.6 \pm 1.4$ & $1.9 \pm 0.4$ & $31.5 \pm 1.1$ & 0.028 & 0.470 & 0.498 \\
\hline Nitrogen & $67.8 \pm 1.4$ & $5.6 \pm 0.6$ & $26.5 \pm 1.1$ & 0.083 & 0.391 & 0.473 \\
\hline
\end{tabular}

significant impregnation of $\mathrm{O}$ groups onto the surface. This suggests that DBD at medium pressure is as efficient as atmospheric pressure systems in raising the oxygen content [29]. The rise in oxygen content values suggests that new oxygen-containing polar groups are formed on the surface of nonwoven textiles. After a certain treatment time, the relative oxygen content on the surface stopped increasing, resulting in a saturated state of wettability. This indicates that, at saturation, the sample surfaces reach a limiting level of oxidation. After plasma treatment, dry air samples resulted in a 134 and $190 \%$ increase in oxygen and nitrogen, respectively, whereas nitrogen samples resulted in a 113 and $560 \%$ increase in oxygen and nitrogen, respectively.

Plasma treatment with dry air produced oxygen-containing functional groups on the surface of polyester samples. The deconvoluted $\mathrm{C} 1 \mathrm{~s}$ peak of untreated and plasma-treated samples is depicted in Figure 6. The $\mathrm{C} 1 \mathrm{~s}$ peak of untreated specimen carries four different peaks at 284.8, 286.1, 286.9,

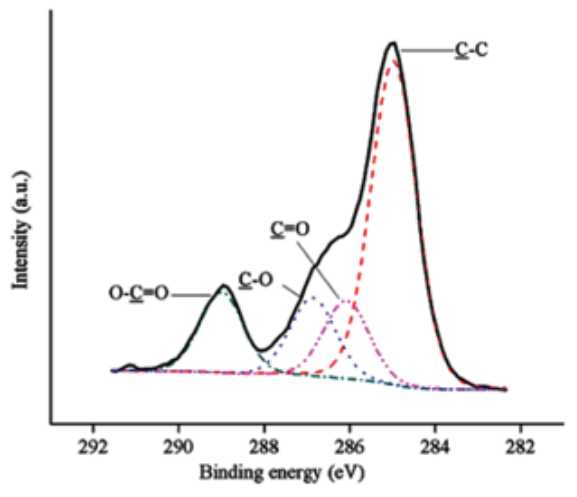

(a) untreated

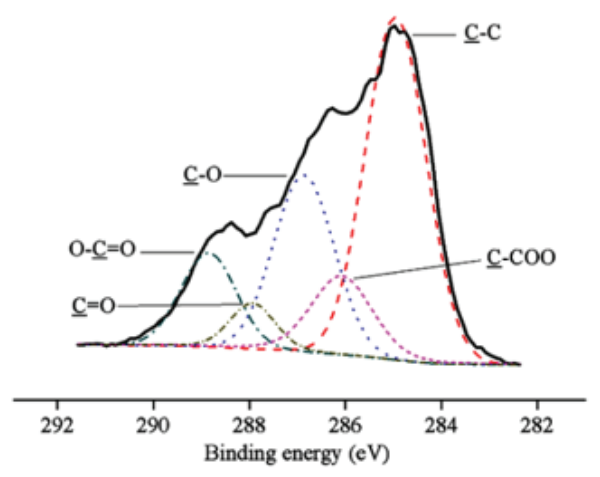

(b) air treated and $288.9 \mathrm{eV}$ corresponding to $\underline{\mathrm{C}}-\mathrm{C}, \underline{\mathrm{C}}=\mathrm{O}, \underline{\mathrm{C}}-\mathrm{O}$, and $\mathrm{O}-\underline{\mathrm{C}}=\mathrm{O}$, respectively, as shown in Figure 6A.

After treatment with air and nitrogen plasma, an additional peak is introduced at $287.8 \mathrm{eV}$ corresponding to $\underline{\mathrm{C}}=\mathrm{O}$ bonds. However, $\underline{\mathrm{C}}=\mathrm{O}$ (amide groups) peak was replaced by $\underline{\mathrm{C}}-\mathrm{COO}$ with $286.1 \mathrm{eV}$ [30]. Therefore, plasma treatment resulted in the formation of a new peak $\underline{\mathrm{C}}-\mathrm{COO}$ with $286.1 \mathrm{eV}$ binding energy as shown in Figure 6B,C. After plasma treatment, the same four components could be found as in the untreated sample. However, also an extra component is seen for the plasma-treated samples because of the air and nitrogen gases. Mostly, the functional groups of oxygen- and nitrogencontaining functionalities appear at similar binding energies [31]. Treatment with nitrogen plasma changes the $\mathrm{O}-\underline{\mathrm{C}}=\mathrm{O}$ and $\underline{\mathrm{C}}-\mathrm{O}$ to nitrogen functional groups $\mathrm{N}-\underline{\mathrm{C}}=\mathrm{O}$ and $\underline{\mathrm{C}}-\mathrm{N}$.

\subsection{Wettability}

3.5.1. Water absorption

Figure 6. C1s curve fit for dry air plasma-activated nonwoven polyester fabric.

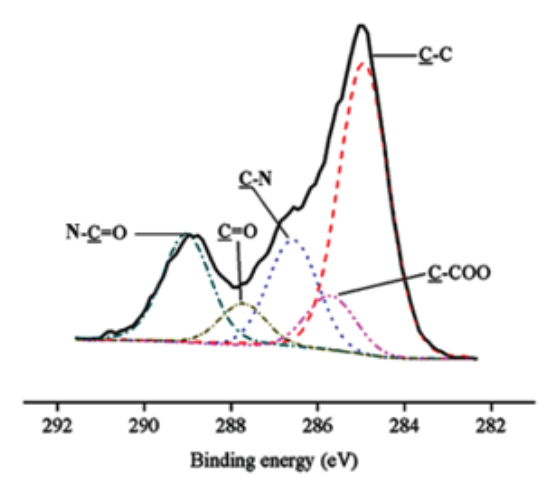

(c) nitrogen treated
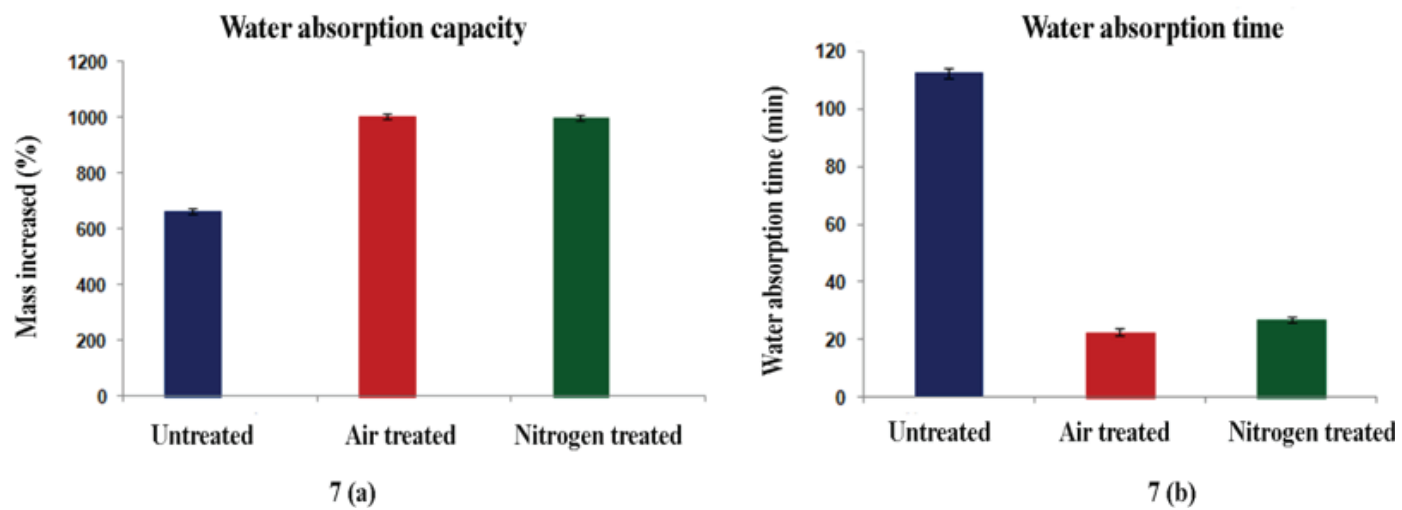

Figure 7. Water absorption. 
Untreated and plasma-treated samples were immersed in water to analyze their water absorption capacity as shown in Figure 7A. It was found that fabric absorption capacity improved by 662 , 1007 , and $1003 \%$ compared to the dry sample for untreated, air-treated, and nitrogen-treated samples, respectively [32]. Plasma-treated samples soaked up more water than the untreated samples after immersing plasma-treated samples in water for $5 \mathrm{~min}$ and hanging them for $5 \mathrm{~s}$.

The water absorption time emphasizes the phenomenon during the first contact of water with the substrate. As the water droplets were poured onto the surface of untreated and plasmatreated fabric samples, no immediate absorption was noticed for all of them; however, after 22 and $27 \mathrm{~min}$, the reflection of droplets disappeared and $100 \%$ wettability of air and nitrogen plasma-treated samples was observed, respectively [32]. In contrast, the droplets on the surface of untreated fabric showed $100 \%$ wettability only after $112 \mathrm{~min}$, as shown in Figure 7B; however, this surface property of the material indicated that the surface energy and interfacial tension observed for treated samples were too low to induce immediate adsorption. Still, a high difference was noted compared to the untreated samples.

Plasma treatment is not permanent but the aging behavior of the air plasma-treated textiles shows the largest aging effect. The limitation of the polar group movement and thus of the aging method will be obtained by cross-linking of the polymer chains throughout plasma treatment. A nonwoven air-plasmatreated textile was not cross-linked and therefore exhibited a substantial aging effect [19].

The capillary rise measurement method has been used to measure the aging characteristics of man-made textile fabrics after 1 week, 1 month, and 6 months' samples. The aging for the air-treated PET fabric samples reported higher than the samples treated with other gasses even 6 months later [33].

\section{Conclusion}

Wetting and hydrophilic characteristics of nonwoven polyester fiber sheets increased after air and nitrogen plasma treatments, caused by the formation of $\mathrm{C}=\mathrm{O}$ and $\mathrm{O}-\mathrm{C}=\mathrm{O}$ polar groups on the surface of the substrate, while inadequate nitrogen-containing groups were observed, as presented in the XPS analysis. WCA of the plasma-treated samples displayed a highly significant difference compared to that of the untreated polyester sample. WCA of the samples decreased to a minimum level and slightly increased after the energy density of 14.92 and $11.21 \mathrm{~J} / \mathrm{cm}^{2}$ for air and nitrogen, respectively, so these values of energy density were fixed for AFM, XPS, and wettability discussion sections. The SEM images indicated the creation of light spots on the surface of nonwoven polyester after DBD treatment, which was further visualized by AFM. AFM showed that specimens treated with air plasma and subjected to etching effects and roughness produced a more, hydrophilic material compared to nitrogen-treated specimens. The XPS analysis verified the incorporation of functional groups on the surface after both DBD oxygen and nitrogen plasma treatments. The XPS analysis is in good agreement with WCA results. In terms of oxygen plasma treatment, significant changes were seen in XPS spectra of the peak C1s with energy density, whereas the changes with nitrogen-plasma treatment were less pronounced. Wetting properties reinforced the results and indicated that the presence of various functional groups on the surface of nonwoven polyester leads to better liquid water collection, which would make the plasma-activated PET nonwovens ideal substrates for hydrophilicity.

Acknowledgment: The authors would like to thank the Technical University of Liberec (TUL) for Student Grant (SGS 21313) 2019.

\section{References}

[1] Gotoh, K., Yasukawa, A., Kobayashi, Y. (2011). Wettability characteristics of poly (ethylene terephthalate) films treated by atmospheric pressure plasma and ultraviolet excimer light. Polymer Journal, 43(6), 545.

[2] DeLassus, P. T., Whiteman, N. F. (2003). Physical and mechanical properties of some important polymers. Wiley Database of Polymer Properties.

[3] Alakara, Ş. F., Karakişla, M., Saçak, M. (2008). Preparation of poly (ethylene terephthalate)-g-Methacrylamide copolymers initiated by azobisizobutyronitrile: Characterization and investigation of some properties. Journal of Macromolecular Science, Part A: Pure and Applied Chemistry, 45(4), 276-280.

[4] Kim, E.-Y., An, S.-K., Kong, J.-S., Kim, H.-D. (2000). Surface modification of polymers and improvement of the adhesion between evaporated copper metal film and a polymer. I. Chemical modification of PET. Journal of Adhesion Science and Technology, 14(9), 1119-1130.

[5] Lee, S. H., Song, W. S. (2010). Surface modification of polyester fabrics by enzyme treatment. Fibers and Polymers, 11(1), 54-59.

[6] Parvinzadeh, M., Ebrahimi, I. (2011). Atmospheric air-plasma treatment of polyester fiber to improve the performance of nanoemulsion silicone. Applied Surface Science, 257(9), 4062-4068.

[7] Siriviriyanun, A., O'Rear, E. A., Yanumet, N. (2007). Modification of polyester fabric properties by surfactant aided surface polymerization. Journal of Applied Polymer Science, 103(6), 4059-4064.

[8] Poll, H., Schladitz, U., Schreiter, S. (2001). Penetration of plasma effects into textile structures. Surface and Coatings Technology, 142, 489-493.

[9] Hegemann, D. (2005). Stain repellent finishing on fabrics. Advanced Engineering Materials, 7(5), 401-404.

[10] Hossain, M., Hegemann, D., Herrmann, A. S., Chabrecek, P. (2006). Contact angle determination on plasma-treated poly (ethylene terephthalate) fabrics and foils. Journal of Applied Polymer Science, 102(2), 1452-1458.

[11] Poll, H.-U., Schreiter, S. (1998). Industrienahe Plasmabehandlung textiler Bahnware. MelliandTextilberichte, 79(6), 466-468.

[12] Hossain, M. M., Herrmann, A. S., Hegemann, D. (2006). Plasma hydrophilization effect on different textile structures. Plasma Processes and Polymers, 3(3), 299-307. 
[13] Bechter, D., et al. (1999). Surface modification of aramid fibers to improve the bond strength through plasma treatment. Technische Textilien, 42, 14-15.

[14] Šimor, M., Ráhel', J., Černák, M., Imahori, Y., Štefečka, M., et al. (2003). Atmospheric-pressure plasma treatment of polyester nonwoven fabrics for electroless plating. Surface and Coatings Technology, 172(1), 1-6.

[15] Pochner, K., Beil, S., Horn, H., Blömer, M. (1997). Treatment of polymers for subsequent metallization using intense UV radiation or plasma at atmospheric pressure. Surface and Coatings Technology, 97(1-3), 372-377.

[16] Kan, C.-W., Yuen, C.-W. M. (2008). Static properties and moisture content properties of polyester fabrics modified by plasma treatment and chemical finishing. Nuclear Instruments and Methods in Physics Research Section B: Beam Interactions With Materials and Atoms, 266(1), 127132.

[17] Sereda, P. J., Feldman, R. (1964). 27-electrostatic charging on fabrics at various humidities. Journal of the Textile Institute Transactions, 55(5), T288-T298.

[18] Kan, C.-W. (2007). Evaluating antistatic performance of plasma-treated polyester. Fibers and Polymers, 8(6), 629634.

[19] Morent, R., De Geytera, N., Verschurenb, J., De Clerckb K., Kiekensb, P., et al. (2008). Non-thermal plasma treatment of textiles. Surface and Coatings Technology, 202(14), 3427-3449.

[20] Cools, P., De Geyter, N., Vanderleyden, E., Dubruel, P., Morent, R. (2014). Surface analysis of titanium cleaning and activation processes: Non-thermal plasma versus other techniques. Plasma Chemistry and Plasma Processing, 34(4), 917-932.

[21] Azeem, M., Wiener, J., Khan, M. Z. (2018). Hydrophobic analysis of nano-filament polyester fabric. Vlákna a Textil, 25(1), 5 .

[22] Öktem, T., Seventekin, N., Ayhan, H., Piskin, E. (1999). Modification of polyester fabrics by in situ plasma or post-plasma polymerisation of acrylic acid. Coloration Technology, 115(9), 274-279.
[23] De Geyter, N., Morent, R., Leys, C. (2006). Surface modification of a polyester non-woven with a dielectric barrier discharge in air at medium pressure. Surface and Coatings Technology, 201(6), 2460-2466.

[24] Vesel, A., Cvelbar, U., Mozetic, M., Junkar, I., Kovač, J. (2008). Surface modification of polyester by oxygen-and nitrogen-plasma treatment. Surface and Interface Analysis: An International Journal Devoted to the Development and Application of Techniques for the Analysis of Surfaces, Interfaces and thin Films, 40(11), 1444-1453.

[25] Tsougeni, K., Tserepi, A., Boulousis, G., Constantoudis, V., Gogolides, E. (2007). Control of nanotexture and wetting properties of polydimethylsiloxane from very hydrophobic to super-hydrophobic by plasma processing. Plasma Processes and Polymers, 4(4), 398-405.

[26] Kan, C.-W. (2015). Effect of nature of gas in plasma treatment on thermomechanical properties of polyester fibres. Fibers and polymers, 16(8), 1696-1704.

[27] Kogelschatz, U. (2003). Dielectric-barrier discharges: Their history, discharge physics, and industrial applications. Plasma Chemistry and Plasma Processing, 23(1), 1-46.

[28] Dai, X., Kviz, L. (2001). Textile Institute 81st World Conference, Melbourne, Australia, April 2001.

[29] Lee, S. H., Cools, P., Yeo, S. Y., Morent, R. (2018). Plasma polymerization onto nonwoven polyethylene/polypropylene fibers for laccase immobilization as dye decolorization filter media. Textile Research Journal, 89(17), 3578-3590.

[30] Sigurdsson, S., Shishoo, R. (1997). Surface properties of polymers treated with tetrafluoromethane plasma. Journal of Applied Polymer Science, 66(8), 1591-1601.

[31] Beamson, G. (1992). High resolution XPS of organic polymers. The Scienta ESCA 300 Database.

[32] Chen, Q., Tang, K.-P. M., Ma, P., Jiang, G. (2016). Evaluation of water absorption and transport properties of weft knitted polyester fabrics by spontaneous uptake water transport tester and conventional test methods. Fibers and Polymers, 17(8), 1287-1295.

[33] Yaman, N., Koçum, I. C., Öktem, T., Özdoğan, E., Ayhan,

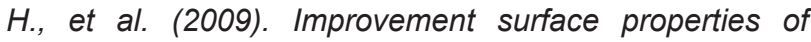
polypropylene and polyester fabrics by glow discharge plasma system under atmospheric condition. Tekstil ve Konfeksiyon, 19(1), 45-51. 